\title{
DISCRETE APPROXIMATION AND CONTROL OF HIGH-ORDER NONLINEAR CONTINUOUS SYSTEMS
}

\author{
Thomas Moor ${ }^{*}$ Jörg Raisch ${ }^{\dagger}$ Alexander Itigin \\ * Research School of Information Sciences and Engineering, \\ Australian National University, Canberra, thomas.moor@anu.edu.au \\ ${ }^{\dagger}$ Lehrstuhl für Systemtheorie technischer Prozesse, Otto-von-Guericke Universität, \\ and Max-Planck-Institut für Dynamik komplexer technischer Systeme, \\ Magdeburg, Germany,raisch@mpi-magdeburg.mpg.de \\ $\$$ Institut für Systemdynamik und Regelungstechnik, \\ Universität Stuttgart, Germany, itigin@isr.uni-stuttgart.de
}

\begin{abstract}
Approximation-based approaches to hybrid control systems synthesis have been mostly limited to problems with low-order linear continuous dynamics. In this contribution, results from the theory of monotone dynamical systems are used to efficiently compute discrete approximations for a class of nonlinear models. Furthermore, a situation is investigated where the high-dimensional plant state converges to a low-dimensional manifold; in the proposed approach the computational effort is governed by the dimension of the loworder manifold without neglecting the high-order dynamics. Results are applied to synthesize a discrete event controller for the automatic start-up of a nonlinear distillation column model of 42nd order. Copyright (C) 2002 IFAC
\end{abstract}

Keywords: hybrid systems, nonlinear systems, monotone systems, discrete event systems, supervisory control.

\section{INTRODUCTION}

A standard approach for synthesizing discrete event controllers for continuous or hybrid systems is based on conservative approximation: by computing a suitable discrete abstraction, the hybrid synthesis problem is transformed into a purely discrete problem, which can subsequently be solved using methods from DES theory. Various approximation based approaches have been suggested, for example, in (Cury et al., 1998; Asarin et al., 2000). The authors of the present paper have proposed $l$-complete approximation (Moor and Raisch, 1999) as a particularly convenient abstraction scheme: by construction, it provides a conservative approximation, i.e. its behaviour includes the underlying system behaviour, and by increasing the parameter $l \in \mathbb{N}_{0}$, approximation accuracy is improved.

In principle, the computational procedure to generate $l$-complete approximations is straightforward. There are, however, two major problems that have limited application of the procedure to a fairly small class of problems: (i) Quantization cells have to be tracked under the progress of time and intersected with other quantization cells. Clearly, this is a difficult problem if the right hand side of the differential or difference equation is nonlinear in the continuous state variable. (ii) Computational effort "explodes" with growing state dimension. Hence, applications have been restricted to fairly low-dimensional plant models.

In this paper, it is shown how the approximation based approach can be extended to a class of high-order nonlinear systems. The paper is organized as follows: in Section 2, the basic procedure for $l$-complete approximation is summarized. For convenience, the discussion is restricted to sampled systems with equidistant sampling instants. Section 3 provides an introduction to monotone dynamical systems and shows how $l$ complete approximations can be computed efficiently 
for this class of nonlinear systems. In Section 4, a situation is explored that allows treatment of highdimensional systems. Finally, in Section 5, the results are applied to synthesize a DES controller for the start-up of a nonlinear distillation column model of order 42 .

\section{APPROXIMATION BASED CONTROL}

The purpose of this section is to state a class of hybrid control problems and to provide references to a general solution procedure. The considered plant is characterized by discrete event inputs and outputs while the internal state is real-valued:

$$
\begin{gathered}
x(k+1)=F(x(k), u(k)), \\
y(k) \in G(x(k)),
\end{gathered}
$$

where $|U| \in \mathbb{N},|Y| \in \mathbb{N}, u: \mathbb{N}_{0} \rightarrow U, y: \mathbb{N}_{0} \rightarrow Y$, $x: \mathbb{N}_{0} \rightarrow \mathbb{R}^{n}, F: \mathbb{R}^{n} \times U \rightarrow \mathbb{R}^{n}, G: \mathbb{R}^{n} \rightarrow 2^{Y} \backslash\{\emptyset\}$. The above system class addresses sampled continuous dynamics with discrete-valued inputs and quantized outputs. By allowing the measurement map $G$ to be nondeterministic, the quantization cells may cover (instead of partition) the continuous state space. This models the practically important case where measurement information is, to a certain extent, ambiguous.

From the perspective of a potential controller, the system has a discrete event behaviour: at the $k$-th sampling instance, the supervisor applies an input symbol $u(k)$ from the finite alphabet $U$ and then waits for the next measurement symbol $y(k+1)$, from the finite alphabet $Y$. However, the external behaviour depends on the continuous dynamics as represented by the next state map $F$. Thus, the considered scenario constitutes a non-trivial class of hybrid systems.

A thorough discussion of supervisory control of hybrid systems is given in (Moor and Raisch, 1999), which is presented within the framework of J.C. WILLEMS' behavioural systems theory; see (Willems, 1991) for a comprehensive introduction. For the scope of this paper, focus attention on the external behaviour $\mathfrak{B} \subseteq(U \times Y)^{\mathbb{N}_{0}}$ induced by the above hybrid plant:

$$
\mathfrak{B}:=\{(u, y) \mid \exists x \forall k:(1) \text { and (2) hold }\} ;
$$

i.e. $\mathfrak{B}$ denotes the set of all trajectories that agree with the plant equations (1) and (2). The task of a supervisor is to restrict the plant behaviour according to a given language inclusion specification: the supervisor shall prevent the system to evolve on trajectories that are deemed to be unacceptable. Formally, the closedloop behaviour $\mathfrak{B}_{\mathrm{cl}}$ is defined as the intersection of $\mathfrak{B}$ with the supervisory controller behaviour $\mathfrak{B}_{\text {sup }}$. The language inclusion specification $\mathfrak{B}_{\text {spec }}$ then demands

$$
\mathfrak{B}_{\mathrm{cl}}:=\mathfrak{B} \cap \mathfrak{B}_{\text {sup }} \subseteq \mathfrak{B}_{\text {spec }} .
$$

If $\mathfrak{B}$ and $\mathfrak{B}_{\text {spec }}$ are both realised by finite state machines, the controller synthesis problem can be treated by a slightly modified version of known methods from DES theory; e.g. (Ramadge and Wonham, 1989). However, for the hybrid plant (1), (2), one cannot expect a finite realisation of $\mathfrak{B}$ to exist. Therefore, it is proposed to base the synthesis of a supervisor $\mathfrak{B}_{\text {sup }}$ on a discrete abstraction of the plant, i.e. a finite automaton realizing a behaviour $\mathfrak{B}_{\mathrm{ca}} \supseteq \mathfrak{B}$. The main result of (Moor and Raisch, 1999) then guarantees that this supervisor not only enforces the specification when connected to the abstraction $\mathfrak{B}_{\text {ca }}$ but also when connected to the actual hybrid plant (1), (2).

In the case of time invariant systems, a particularly suitable abstraction is the l-complete approximation $\mathfrak{B}_{l} \supseteq \mathfrak{B}$, where $l \in \mathbb{N}$ is a parameter. The most relevant features of $\mathfrak{B}_{l}$ are that (i) accuracy is monotone in $l$, i.e. $\mathfrak{B}_{l+1} \subseteq \mathfrak{B}_{l}$, and that (ii) a finite realization can be easily derived from the restricted plant behaviour $\left.\mathfrak{B}\right|_{[0, l]}$. The following iterative procedure is recalled from (Moor and Raisch, 1999):

Theorem 1. Let $\mathfrak{B} \subseteq(U \times Y)^{\mathbb{N}_{0}}$ denote the external behaviour of $(1),(2)$. For $(u, y) \in(U \times Y)^{\mathbb{N}_{0}}$ and $l \in \mathbb{N}_{0}$ define the sets of compatible states $\mathcal{X}\left(\left.(u, y)\right|_{[0, l]}\right) \subseteq \mathbb{R}^{n}$ iteratively by

$$
\begin{aligned}
& \mathcal{X}\left(\left.(u, y)\right|_{[0,0]}\right):=G^{-1}(y(0)), \\
& \mathcal{X}\left(\left.(u, y)\right|_{[0, l+1]}\right):= \\
& \quad F\left(\mathcal{X}\left(\left.(u, y)\right|_{[0, l]}\right), u(l)\right) \cap G^{-1}(y(l+1)) .
\end{aligned}
$$

Then

$$
\left.\left.(u, y)\right|_{[0, l]} \in \mathfrak{B}\right|_{[0, l]} \Leftrightarrow \mathcal{X}\left(\left.(u, y)\right|_{[0, l]}\right) \neq \varnothing .
$$

By the above theorem, $\left.\mathfrak{B}\right|_{[0, l]}$ can be established via a finite iteration of images under $F$ and intersections with the quantization cells $G^{-1}$. Then, the methods presented in (Moor and Raisch, 1999) allow the construction of a finite abstraction of the hybrid plant and finally the synthesis of a supervisory controller.

From a practical perspective, however, there are two major limitations. First, for nonlinear continuous dynamics, images of sets of states under $F$ can, in general, not be computed efficiently. Roughly speaking, one is left with the simulation from an exhaustive number of initial conditions $x(0)$, and then naively assumes that $\mathcal{X}\left(\left.(u, y)\right|_{[0, l]}\right)=\emptyset$ whenever no witness $\xi \in \mathcal{X}\left(\left.(u, y)\right|_{[0, l]}\right)$ could be found, at the risk of violating the requirement $\mathfrak{B}_{\mathrm{ca}} \supseteq \mathfrak{B}$. Second, for high dimensional continuous dynamics, a reasonably accurate quantization leads to computationally intractable output alphabets $Y$. In the following two sections, a broad class of hybrid systems is identified where the above iterative procedure can be refined in order to gain substantial computational efficiency.

\section{MONOTONE DYNAMICAL SYSTEMS}

For monotone dynamical systems (see (Smith, 1995) for a comprehensive treatment of the subject), it is 
possible to efficiently estimate the sets of compatible states $\mathcal{X}\left(\left.(u, y)\right|_{[0, l]}\right)$. In general, monotonicity is defined with respect to an arbitrary partial order. The scope of this paper, however, is restricted to the specific partial order $\preccurlyeq$, where, for $a, b \in \mathbb{R}^{n}$,

$$
a \preccurlyeq b: \Leftrightarrow a_{i} \leq b_{i}, \quad i=1, \ldots n .
$$

Definition 2. The map $g: \mathbb{R}^{q} \rightarrow \mathbb{R}^{n}$ is called order preserving if $a \preccurlyeq b$ implies $g(a) \preccurlyeq g(b)$.

Note that a map is order preserving if all its partial derivatives are nonnegative. The image of a "box"

$$
Q(a, b):=\{c \mid a \preccurlyeq c \preccurlyeq b\}
$$

under an order preserving map $g$ can be efficiently over-approximated via the images of $a$ and $b$, i.e. $g(Q(a, b)) \subseteq Q(g(a), g(b))$.

For the following consider dynamical systems

$$
\dot{z}(t)=f(z(t))
$$

and assume that for any initial condition $z(0)=z_{0}$ there exists a unique solution $\Phi_{t}\left(z_{0}\right)$ for all $t \geq 0$. The system (10) is called monotone, if ordered states remain ordered under the progress of time, i.e. if the flow $\Phi_{t}: \mathbb{R}^{n} \rightarrow \mathbb{R}^{n}$ induced by the vector field $f: \mathbb{R}^{n} \rightarrow \mathbb{R}^{n}$ is order preserving for all $t \geq 0$.

A criterion for monotonicity can be stated in terms of the off-diagonal entries of the Jacobian of $f$ :

Theorem 3. (see e.g. (Smith, 1995)) The dynamical system $\dot{z}=f(z)$ is monotone if

$$
\partial f_{i} / \partial z_{j} \geq 0, \quad \forall i \neq j
$$

For monotone systems, the temporal evolution of a box $Q\left(\zeta_{a}, \zeta_{b}\right)$ can be over-approximated by evaluating the flow for the points $\zeta_{a}$ and $\zeta_{b}$ only: $\Phi_{t}\left(Q\left(\zeta_{a}, \zeta_{b}\right)\right) \subseteq$ $Q\left(\Phi_{t}\left(\zeta_{a}\right), \Phi_{t}\left(\zeta_{b}\right)\right)$. In the case of the hybrid system (1), (2), the continuous dynamics depends on a fixed control symbol $\mu \in U$, i.e. $\dot{z}=f_{\mu}(z)$. As before, it is assumed that the flow $\Phi_{t}^{\mu}: \mathbb{R}^{n} \rightarrow \mathbb{R}^{n}$ induced by $f_{\mu}$ exists for all $t \geq 0$ and is order preserving. Hence, the transition function defined for a fixed sampling interval $\Delta>0$,

$$
F(\xi, \mu):=\Phi_{\Delta}^{\mu}(\xi),
$$

is also order preserving.

It is further assumed that measurement symbols $v_{j}$, $j=1, \ldots, p$, correspond to bounded boxes in $\mathbb{R}^{n}$, i.e.

$$
G^{-1}\left(v_{j}\right)=Q\left(a_{j}, b_{j}\right),
$$

where $a_{j}, b_{j} \in \mathbb{R}^{n}, a_{j} \preccurlyeq b_{j}$.

Obviously, a finite number of boxes (13) cannot cover the entire $\mathbb{R}^{n}$. Hence, one needs an additional "out of range symbol" \& with

$$
G^{-1}(\$)=\mathbb{R}^{n} \backslash \cup_{1 \leq j \leq p} G^{-1}\left(v_{j}\right)
$$

to give $Y=\left\{v_{1}, \ldots, v_{p}\right\} \cup\left\{\begin{array}{l}\text { f } \\ \text {. }\end{array}\right.$
Based on the iteration (5), (6) and on $\hat{F}(Q(a, b), \mu):=$ $Q(F(a, \mu), F(b, \mu))$ as an over-approximation of the continuous evolution of a box $Q(a, b)$ under the flow $\Phi_{\Delta}^{\mu}$, easily computable safe estimates $\hat{\mathcal{X}}\left(\left.(u, y)\right|_{[0 . l]} \subseteq\right.$ $\mathbb{R}^{n}$ for the sets of compatible states are provided as follows:

$$
\begin{aligned}
& \text { if } y(0)=v_{j} \neq \ddagger \text { for some } j \text {, let } \\
& \hat{\mathcal{X}}\left(\left.(u, y)\right|_{[0,0]}\right):=G^{-1}\left(v_{j}\right) ; \\
& \text { if } y(0)=t \text {, let } \\
& \hat{\mathcal{X}}\left(\left.(u, y)\right|_{[0,0]}\right):=\mathbb{R}^{n} ; \\
& \text { if } y(l+1) \neq t \text { and } \hat{\mathcal{X}}\left(\left.(u, y)\right|_{[0, l]}\right) \neq \mathbb{R}^{n} \text {, let } \\
& \hat{\mathcal{X}}\left(\left.(u, y)\right|_{[0 . l+1]}\right):= \\
& \hat{F}\left(\hat{\mathcal{X}}\left(\left.(u, y)\right|_{[0, l]}\right), u(l)\right) \cap G^{-1}(y(l+1)) ; \\
& \text { if } y(l+1) \neq \ddagger \text { and } \hat{\mathcal{X}}\left(\left.(u, y)\right|_{[0, l]}\right)=\mathbb{R}^{n} \text {, let } \\
& \hat{\mathcal{X}}\left(\left.(u, y)\right|_{[0 . l+1]}\right):=G^{-1}(y(l+1)) ; \\
& \text { if } y(l+1)=\hbar \text { and } \hat{\mathcal{X}}\left(\left.(u, y)\right|_{[0, l]}\right) \neq \mathbb{R}^{n} \text { and } \\
& \hat{F}\left(\hat{\mathcal{X}}\left(\left.(u, y)\right|_{[0, l]}\right), u(l)\right) \nsubseteq \cup_{1 \leq j \leq p} G^{-1}\left(v_{j}\right) \text {, let } \\
& \hat{\mathcal{X}}\left(\left.(u, y)\right|_{[0, l+1]}\right):=\hat{F}\left(\hat{\mathcal{X}}\left(\left.(u, y)\right|_{[0, l]}\right), u(l)\right) ; \\
& \text { if } y(l+1)=\ddagger \text { and } \hat{\mathcal{X}}\left(\left.(u, y)\right|_{[0, l]}\right) \neq \mathbb{R}^{n} \text { and } \\
& \hat{F}\left(\hat{\mathcal{X}}\left(\left.(u, y)\right|_{[0 . l]}\right), u(l)\right) \subseteq \cup_{1 \leq j \leq p} G^{-1}\left(v_{j}\right) \text {, let } \\
& \hat{\mathcal{X}}\left(\left.(u, y)\right|_{[0, l+1]}\right):=\emptyset \\
& \text { if } y(l+1)=t \text { and } \hat{\mathcal{X}}\left(\left.(u, y)\right|_{[0, l]}\right)=\mathbb{R}^{n} \text {, let } \\
& \hat{\mathcal{X}}\left(\left.(u, y)\right|_{[0, l+1]}\right):=\mathbb{R}^{n} \text {. }
\end{aligned}
$$

Iteration (15)-(21) is well-defined; in particular, the sets $\hat{\mathcal{X}}\left(\left.(u, y)\right|_{[0, l+1]}\right)$ are either $\mathbb{R}^{n}$ or bounded boxes in $\mathbb{R}^{n}$. From $\hat{F}(Q(a, b), \mu) \supseteq F(Q(a, b), \mu)$, it follows that $\hat{\mathcal{X}}\left(\left.(u, y)\right|_{[0, l]}\right) \supseteq \mathcal{X}\left(\left.(u, y)\right|_{[0, l]}\right)$. Therefore, $\left.\hat{\mathfrak{B}}\right|_{[0, l]}:=\left\{\left.(u, y)\right|_{[0, l]}\left|\hat{\mathcal{X}}\left(\left.(u, y)\right|_{[0, l]} \neq \emptyset\right\} \supseteq \mathfrak{B}\right|_{[0, l]}\right.$ forms a suitable basis for controller synthesis.

\section{HANDLING HIGH-ORDER DYNAMICS}

Many complex technical processes, although intrinsically high-dimensional, converge to a low-dimensional manifold within a short time; see the distillation column scenario in Sec. 5. Or, one may design a number of low-level continuous controllers such that each individual continuous closed-loop exhibits an attractive low-dimensional manifold, even if the plant itself does not do so. In the hierarchical control architecture discussed in (Moor et al., 2001), a highlevel supervisor then switches between the low-level controllers, in order to enforce an overall specification.

This particular structure can be exploited in the following way: instead of quantizing the high-dimensional plant state space, only a well defined neighbourhood of the relevant part of the respective manifold 
is covered by quantization cells and hence provides measurement information; the "rest" of the state space returns an "out of range" symbol. For a formal treatment of this idea, let

$$
h_{\mu}: \mathbb{R}^{q} \rightarrow \mathbb{R}^{n}, q<n,
$$

represent a parametrization of a $q$-dimensional manifold $\mathcal{M}_{\mu}$ in $\mathbb{R}^{n}$. Naturally, both the manifold and its parametrization may depend on the control symbol $\mu$. Assume $h_{\mu}$ to be order preserving and $\mathcal{M}_{\mu}$ to be attractive, i.e.

$$
\lim _{t \rightarrow \infty} \operatorname{dist}\left(h_{\mu}\left(\mathbb{R}^{q}\right), z(t)\right)=0
$$

where $^{1} \operatorname{dist}(X, \zeta):=\inf \left\{\|\zeta-\xi\|_{\infty}^{\beta} \mid \xi \in X\right\}$.

Let the bounded subset $P \subset \mathbb{R}^{q}$ represent the relevant operating range on $\mathcal{M}_{\mu}$ and

$$
\mathcal{V}_{\delta}\left(h_{\mu}(P)\right):=\left\{\zeta \mid \operatorname{dist}\left(h_{\mu}(P), \zeta\right)<\delta\right\}
$$

the neighbourhood of $h_{\mu}(P)$ that is to be covered by quantization cells. This can be achieved by covering $P$ with a number of ( $q$-dimensional) boxes and suitably "inflating" their images under $h_{\mu}$ :

$$
\begin{gathered}
P \subseteq \cup_{1 \leq j \leq p_{\mu}} Q\left(a_{j}, b_{j}\right), \quad a_{j}, b_{j} \in \mathbb{R}^{q}, \\
G^{-1}\left(v_{j}^{\mu}\right):=Q\left(h_{\mu}\left(a_{j}\right)-\delta \beta^{-1}, h_{\mu}\left(b_{j}\right)+\delta \beta^{-1}\right),
\end{gathered}
$$

where $\beta^{-1}:=\left(\beta_{1}^{-1}, \ldots \beta_{n}^{-1}\right)^{\boldsymbol{\top}}$. Then, as required,

$$
\cup_{1 \leq j \leq p_{\mu}} G^{-1}\left(v_{j}^{\mu}\right) \supseteq \mathcal{V}_{\delta}\left(h_{\mu}(P)\right) .
$$

To prove (27), take any $\zeta \in \mathcal{V}_{\delta}\left(h_{\mu}(P)\right)$. Then there exists a $\hat{p} \in P, \hat{\zeta}:=h_{\mu}(\hat{p})$, such that $\|\hat{\zeta}-\zeta\|_{\infty}^{\beta} \leq \delta$, and therefore $\hat{\zeta}-\delta \beta^{-1} \preccurlyeq \zeta \leqslant \hat{\zeta}+\delta \beta^{-1}$. By (25), one can find a $j$ such that $\hat{p} \in Q\left(a_{j}, b_{j}\right)$, i.e. $a_{j} \preccurlyeq \hat{p} \preccurlyeq$ $b_{j}$. As $h_{\mu}$ is order preserving, $h_{\mu}\left(a_{j}\right) \preccurlyeq h_{\mu}(\hat{p})=\hat{\zeta} \preccurlyeq$ $h_{\mu}\left(b_{j}\right)$. Therefore $h_{\mu}\left(a_{j}\right)-\delta \beta^{-1} \preccurlyeq \zeta \preccurlyeq h_{\mu}\left(b_{j}\right)+$ $\delta \beta^{-1}$ and $\zeta \in G^{-1}\left(v_{j}^{\mu}\right)$.

The part of $\mathbb{R}^{n}$ not covered by any of the cells $G^{-1}\left(\nu_{j}^{\mu}\right)$, returns an "out of range symbol" $t^{\mu}$, i.e.

$$
G^{-1}\left(\dagger^{\mu}\right):=\mathbb{R}^{n} \backslash \cup_{1 \leq j \leq p_{\mu}} G^{-1}\left(v_{j}^{\mu}\right),
$$

such that the set of measurement symbols is given by

$$
Y:=\cup_{\mu \in U} Y_{\mu}, \quad Y_{\mu}:=\left\{v_{1}^{\mu}, \ldots, v_{p_{\mu}}^{\mu}\right\} \cup\left\{\dagger^{\mu}\right\} .
$$

Assuming monotonicity of the high dimensional continuous dynamics, sets of compatible states can again be safely estimated by the iteration (15)-(21). Note that the discrete abstraction based on $\left.\hat{\mathfrak{B}}\right|_{[0 . l]}$ is conservative even if the convergence (23) fails to hold true.

\section{START-UP OF A DISTILLATION COLUMN}

Consider a distillation column in pilot plant scale, which is operated at the Institut für Systemdynamik

\footnotetext{
1 The weighted infinity norm is defined by $\|\xi\|_{\infty}^{\beta}:=\max _{i}\left|\beta_{i} \xi_{i}\right|$. The weighing vector $\beta=\left(\beta_{1} \ldots \beta_{n}\right), \beta_{i}>0, \sum \beta_{i} / n=1$, is assumed to be fixed for the scope of this paper.
}

und Regelungstechnik in Stuttgart. It is about $10 \mathrm{~m}$ high, and consists of 40 bubble cap trays (consecutively numbered by $i=2, \ldots, 41$ from bottom to top), a reboiler $(i=1)$ and a condenser $(i=42)$, see Fig. 1 . Feed is supplied on tray 21. Our application example is the separation of methanol and propanol.

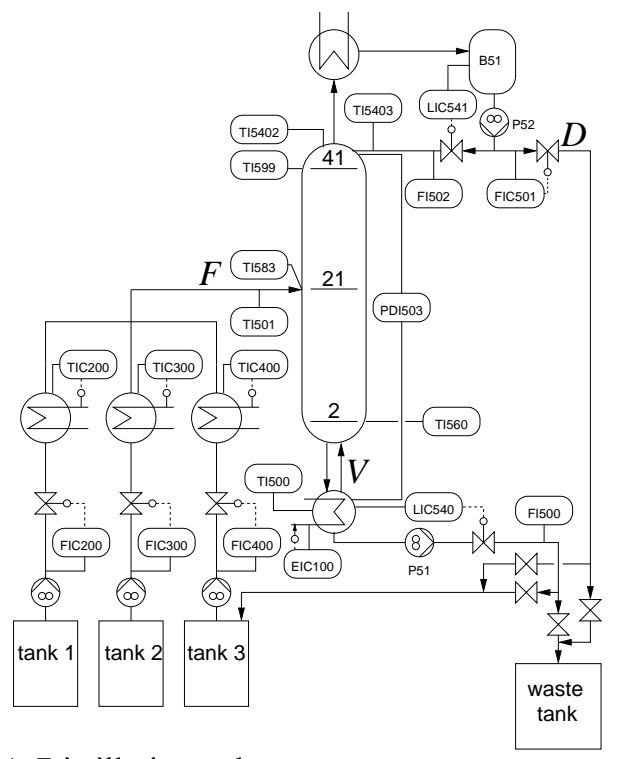

Fig. 1. Distillation column.

The following steps can be distinguished during conventional column start-up: initially, the column trays are partially filled with liquid mixture from the previous experimental run. Further feed is added, and the column is heated up until boiling point conditions are established in the whole column. During this start-up step, the column is operated at total reflux and reboil. At the end of this step, a single concentration front is established. In a second step, the feed, distillate and vapour flow rates are adjusted to their desired steady state values, and the initial front splits into two fronts. Then, in a third step, the two fronts move very slowly towards their steady state.

In the following, the objective is to speed up the third step of the start-up procedure by introducing a suitable supervisory control strategy. The starting point for an approximation based controller synthesis is the below continuous distillation column plant model which incorporates the following assumptions, which are well justified during the third step of start-up: (1) constant molar overflows, (2) constant molar liquid holdups, (3) negligible vapour holdups, (4) total condenser, (5) constant relative volatilities, (6) a tray efficiency of one. Therefore, the model is based on material balances only and consists of one nonlinear first-order ODE for each tray, the reboiler, and the condenser:

$$
\begin{aligned}
n_{L}^{i} \dot{x}_{i} & =F_{L}^{i+1} x_{i+1}-F_{L}^{i} x_{i} \\
& +F_{V}^{i-1} y_{i-1}-F_{V}^{i} y_{i}+ \begin{cases}F x^{F} & \text { if } i=21, \\
0 & \text { else, }\end{cases} \\
y_{i} & =x_{i} \alpha\left(1+x_{i}(\alpha-1)\right)^{-1},
\end{aligned}
$$


where $x_{i}$ and $y_{i}$ are the methanol mole fractions in the liquid and in the vapour on the $i$-th tray, in the condenser $(i=42)$ and the reboiler $(i=1) ; \alpha=$ 2.867 is the relative volatility; $x^{F}=0.32$ is the methanol mole fraction in the feed; $F_{L}^{i}$ denotes the liquid molar flow rate, $F_{V}^{i}$ the vapour flow rate and $n_{L}^{i}$ the molar liquid holdup; $F, D$ and $V$ denote the feed, distillate and vapour flow rate, respectively; See Table 1 for dependencies between the above variables.

Table 1. Flow rates and liquid holdups.

\begin{tabular}{|l|c|c|c|c|c|c|}
\hline & $i$ & $F_{L}^{i+1}$ & $F_{L}^{i}$ & $F_{V}^{i-1}$ & $F_{V}^{i}$ & $n_{L}^{i}$ [mol] \\
\hline condenser & 42 & 0 & $V$ & $V$ & 0 & 1.922 \\
& $22-41$ & $V-D$ & $V-D$ & $V$ & $V$ & 1.922 \\
feed tray & 21 & $V-D$ & $F+V-D$ & $V$ & $V$ & 1.922 \\
& $2-20$ & $F+V-D$ & $F+V-D$ & $V$ & $V$ & 1.922 \\
reboiler & 1 & $F+V-D$ & $F-D$ & 0 & $V$ & 135 \\
\hline
\end{tabular}

The feed flow rate is considered to be constant at $F=220.0[\mathrm{~mol} / \mathrm{h}]$, while $D$ and $V$ are control inputs. For any constant $D$ and $V$, the system (30), (31) has an attractive equilibrium $x^{*}(D, V)$, which, for the nominal inputs $D_{0}=70.4[\mathrm{~mol} / \mathrm{h}]$ and $V_{0}=$ $188.2[\mathrm{~mol} / \mathrm{h}$ ], corresponds to the desired operating point $x_{0}^{*}:=x^{*}\left(D_{0}, V_{0}\right)$ of the distillation column. The controller under construction shall switch between a finite number of constant input values $V$ and $D$. Considering only values $V>0, D>0$ such that $F+V-D \geq 0$, monotonicity of (30), (31) follows from the criterion given in Theorem 3 .

The construction of lower dimensional manifolds $\mathcal{M}_{\mu}$ is based on wave propagation theory; it considers particular concentration profiles as waves and discusses their propagation in time and space (Kienle, 2000). Each wave is of the form

$$
x_{i}=p_{1}+\frac{p_{2}-p_{1}}{1+e^{\rho(i-s)}},
$$

where $p_{1}$ and $p_{2}$ are the asymptotic values of the methanol mole fraction at the bottom and at the top of the wave, $s$ is the so called wave position (point of inflexion) and $\rho$ is the slope at $s$. The outcome of wave propagation theory most relevant to this paper is that during the third startup step, the concentration profile can be represented by two waves of the type (32), one in each the stripping $(1 \leq i \leq 21)$ and rectifying section $(21<i \leq 42)$. Their slopes can be approximated reasonably well by the slopes that correspond to the equilibrium $x_{0}^{*}$, numerical values being $\rho_{s}=0.465$ (stripping section) and $\rho_{r}=0.572$ (rectifying section). Neglecting the effect of different inputs to the slopes, $\mathcal{M}_{\mu}$ becomes independent of $\mu$. Under the additional assumption of constant methanol mole fractions in the reboiler and condenser, $x_{1}=0$ and $x_{42}=1$, the asymptotic values in Eq. (32) are uniquely determined by the feed concentration $x_{21}$ and the wave positions $\hat{s}_{s}$ and $\hat{s}_{r}$ for the stripping and rectifying section, respectively ${ }^{2}$. Consequently,

2 Here, Eq. (32) is re-parametrised for the stripping and rectifying section by $s=22-\hat{s}_{s}$ and $s=63-\hat{s}_{r}$, respectively. the wave fronts of interest are parametrized by a map $h: \mathbb{R}^{3} \rightarrow \mathbb{R}^{42}$ mapping parameter triples $\left(x_{21}, \hat{s}_{S}, \hat{s}_{r}\right)$ to concentration profiles. The $i$-th component $h_{i}$ of $h$ evaluates to

$$
\begin{array}{r}
h_{i}\left(x_{21}, \hat{s}_{s}, \hat{s}_{r}\right):=x_{21}\left[\left(1-e^{(i-1) \rho_{s}}\right)\left(1+e^{\left(\hat{s}_{s}-1\right) \rho_{s}}\right)\right. \\
] \times\left[\left(1-e^{20 \rho_{s}}\right)\left(1+e^{\left(i-22+\hat{s}_{s}\right) \rho_{s}}\right)\right]^{-1}
\end{array}
$$

for $1 \leq i \leq 21$, and

$$
\begin{aligned}
& h_{i}\left(x_{21}, \hat{s}_{S}, \hat{s}_{r}\right):=\left[x_{21}\left(e^{21 \rho_{r}}-e^{\left(i-63+\hat{s}_{r}\right) \rho_{r}}\right)\right. \\
& +\left(1-x_{21}\right)\left(e^{\left(\hat{s}_{r}-21\right) \rho_{r}}-e^{(i-21) \rho_{r}}\right)+e^{\left(i-42+\hat{s}_{r}\right) \rho_{r}} \\
& \quad-1] \times\left[\left(e^{21 \rho_{r}}-1\right)\left(e^{\left(i-63+\hat{s}_{r}\right) \rho_{r}}+1\right)\right]^{-1}
\end{aligned}
$$

for $22 \leq i \leq 42$. Note that all partial derivatives of $h$ are non-negative. Hence, $h$ is order preserving. This completes the construction of $\mathcal{M} \equiv \mathcal{M}_{\mu}:=h\left(\mathbb{R}^{3}\right)$.

The operating range of the supervisor is first given in the parameter space and then lifted to the highdimensional state space. In the particular setting, the equilibrium $x_{0}^{*}$ corresponds to the parameter triple $x_{21} \approx 0.318, \hat{s}_{s} \approx 10.7, \hat{s}_{r} \approx 28.7$. The bounded box of parameters $P=[0.300,0.340] \times[4.0,20.0] \times$ $[23.0,37.0]$ is considered a reasonably large operation range. The high-dimensional measurement quantization is obtained from a partition of $P$ by $p=139$ parameter cells $Q\left(a_{j}, b_{j}\right), 1 \leq j \leq p$ and Eq. (26), where $\delta=0.002$. Input symbols $U=\left\{\mu_{1}, \ldots \mu_{9}\right\}$ are chosen according to Table 2; see (Klein et al., 1999) for a motivation of the particular numerical values. For each $\mu \in U$, the system (30), (31) induces a flow $\Phi_{t}^{\mu}$. Finally, a sampling time of $\Delta=10 \mathrm{~min}$ constitutes a hybrid model according to Sec. 2 .

Table 2. Control symbols.

\begin{tabular}{|c|c|c|}
\hline$D[\mathrm{~mol} / \mathrm{h}]$ & $V[\mathrm{~mol} / \mathrm{h}]$ & control symbol \\
\hline 35.8070 & 188.2433 & $\mu_{1}$ \\
59.3318 & 158.6412 & $\mu_{2}$ \\
82.8566 & 129.0391 & $\mu_{3}$ \\
46.8782 & 217.8455 & $\mu_{4}$ \\
70.4030 & 188.2433 & $\mu_{5}$ \\
93.9278 & 158.6412 & $\mu_{6}$ \\
57.9494 & 247.4476 & $\mu_{7}$ \\
81.4742 & 217.8455 & $\mu_{8}$ \\
104.999 & 188.2433 & $\mu_{9}$ \\
\hline
\end{tabular}

As a specification, the supervisor is required to drive any initial state within $X_{0}=V_{\delta}(h(P))$ into the target region $X_{f}=\mathcal{V}_{\delta}\left(h\left(P_{f}\right)\right)$ within no more than 20min, where $P_{f}=[0.316,0.320] \times[8.5,11.5] \times$ $[27.5,31.0] \subset P$. Choosing one of the quantisation cells equal to $X_{f}$, this specification can easily be formalized as a language inclusion (4). Synthesis is then successfully carried out based on the estimate sets of compatible states $\hat{\mathcal{X}}\left(\left.(u, y)\right|_{[0, l]}\right)$ for $l=1,2$. A closed-loop simulation is shown in Fig. 2. For each sampling instant, one concentration profile is plotted, the arrows indicating forward evolution in time. As the sampling intervals in the closed-loop configuration are chosen to be $10 \mathrm{~min}$, the target region is seen to be reached within $20 \mathrm{~min}$. In contrast, Fig. 3 shows an open-loop simulation for the nominal input $V_{0}$ and $D_{0}$. 


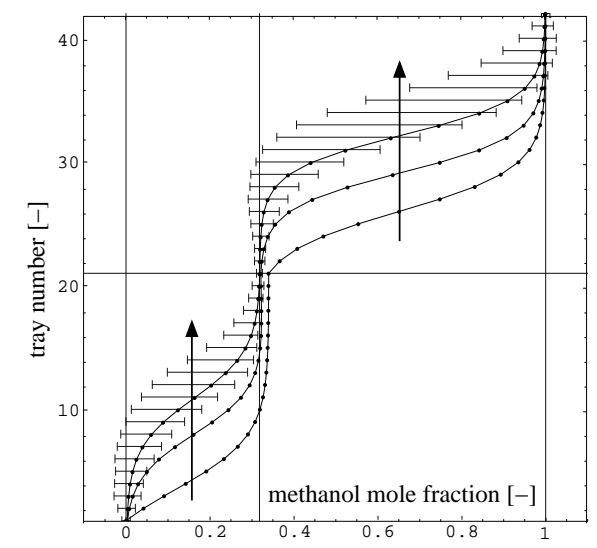

Fig. 2. Closed-loop simulation $(\Delta=10 \mathrm{~min})$

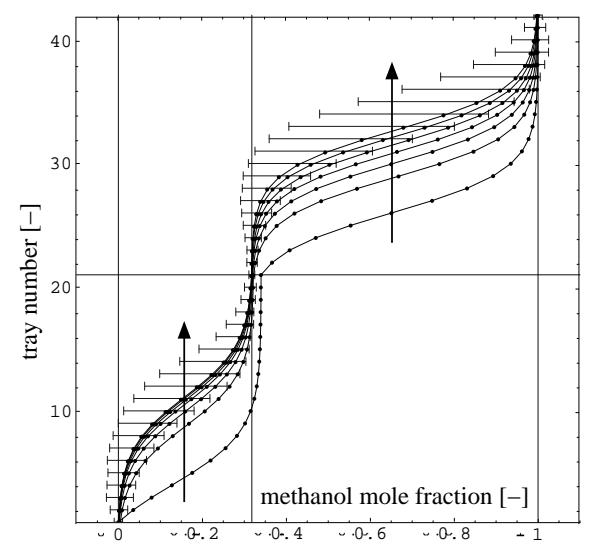

Fig. 3. Open-loop simulation $(\Delta=5 \mathrm{~h})$

Here, one profile every $5 \mathrm{~h}$ is plotted, and it takes an overall time of $20 \mathrm{~h}$ to reach the target region.

Remark: The properties employed for the construction of $\mathcal{M}$ are well motivated by wave propagation theory and also have been validated by simulations and experiments. It follows from the successful completion of the controller synthesis procedure, that our abstraction is accurate enough for the particular purpose. While the insight from the process engineering perspective has been an essential guidance, it is important to note that the reliability of our controller does not depend on the various claims and assumptions regarding the process model: the only relevant requirement is for the approximation to be conservative, i.e. $\mathfrak{B}_{\mathrm{ca}} \supseteq \mathfrak{B}$, and this follows purely from the monotonicity of $f$ and $h$, as discussed in Sec. 3 .

On a decent workstation, the overall time required for the computation of both the discrete approximation and the supervisory controller is about $10 \mathrm{~min}$. This is a significant performance increment, when compared with earlier work (Klein et al., 1999) on the very same scenario, but based on exhaustive simulation: there, computations used to take up multiple hours, depending on the number of considered initial states $x(0) \in \mathbb{R}^{n}$. Note also the different quality of reliability: while our new approach guarantees the approximation to be conservative, exhaustive simulation may -in principle-overlook "most critical" states.

\section{CONCLUSIONS}

This paper discusses approximation based synthesis of discrete event controllers for high-order monotone systems. Here, monotonicity allows for an efficient over-approximation of the temporal evolution of quantization cells, from which conservative discrete approximations of the nonlinear dynamics can be derived. It is also shown how to overcome the problem of high state dimensionality if the plant state approaches a low-dimensional manifold. These contributions are illustrated by an example from process control: based on a 42nd order nonlinear model, a discrete controller is synthesized for the start-up of a pilot plant scale distillation column. A comparison with earlier work underlines the achieved computational benefits.

Acknowledgement: The financial support by the US Office of Naval Research, Grant N 00014-98-1-0535, is gratefully acknowledged. We would also like to thank D. Flockerzi and A. Kienle from MPI Magdeburg for valuable discussions on monotone dynamical systems and the distillation column scenario.

\section{REFERENCES}

Asarin, E., O. Bournez, T. Dang, O. Maler, and A. Pnueli (2000). Effective synthesis of switching controllers for linear systems. Proceedings of the IEEE 88, 1011-1025.

Cury, J.E.R., B.A. Krogh and T. Niinomi (1998). Synthesis of supervisory controllers for hybrid systems based on approximating automata. IEEE Transactions on Automatic Control, Special issue on hybrid systems $\mathbf{4 3}, 564-568$.

Kienle, A. (2000). Low-order models for ideal multicomponent distillation processes using nonlinear wave propagation theory. Chemical Engineering Science 55, 1817-1828.

Klein, E., A. Kienle, J. Raisch and H. Wehlan (1999). Synthese einer Anfahrregelung für eine Destillationskolonne auf der Grundlage einer ereignisdiskreten Approximation der kontinuierlichen Dynamik. 6. Fachtagung Entwicklung and Betrieb komplexer Automatisierungssysteme (EKA99) pp. 447-464.

Moor, T. and J. Raisch (1999). Supervisory control of hybrid systems within a behavioural framework. Systems and Control Letters 38, 157-166.

Moor, T., J. Raisch and J.M. Davoren (2001). Computational advantages of a two-level hybrid control architecture. In: The 40th IEEE CDC, Conference Proceedings. IEEE Press. pp. 358-362.

Ramadge, P.J. and W.M. Wonham (1989). The control of discrete event systems. Proceedings of the IEEE 77, 81-98.

Smith, H.L. (1995). Monotone Dynamical Systems. American Mathematical Society. Providence.

Willems, J.C. (1991). Paradigms and puzzles in the theory of dynamic systems. IEEE Transactions on Automatic Control 36:3, 258-294. 